\title{
Neuropaludism in Children: Clinical and Evolving Aspects in a Prefectoral Hospital in Guinea
}

\author{
Vamala Guilavogui ${ }^{1}$, Nestor Onikoyamou ${ }^{1}$, Seylan Diawara ${ }^{1}$, Kemoko Camara ${ }^{2}$, \\ Foksouna Sakadi ${ }^{3,}$, Kezely Beavogui ${ }^{1}$ \\ ${ }^{1}$ Neurosurgery Department, Donka National Hospital, Conakry, Guinea \\ ${ }^{2}$ Neurology Department, Sino-guinean Hospital, Conakry, Guinea \\ ${ }^{3}$ Neurology Unit, National Reference Teaching Hospital, Ndjamena, Chad \\ Email address: \\ vamalagui@yahoo.fr (V. Guilavogui), foromo1983@gmail.com (N. Onikoyamou), seylandiaw@gmail.com (S. Diawara), \\ ksmfcamara@hotmail.fr (K. Camara), fokasaka@gmail.com (F. Sakadi), drkezely@gmail.com (K. Beavogui) \\ ${ }^{*}$ Corresponding author
}

\section{To cite this article:}

Vamala Guilavogui, Nestor Onikoyamou, Seylan Diawara, Kemoko Camara, Foksouna Sakadi, Kezely Beavogui. Neuropaludism in Children: Clinical and Evolving Aspects in a Prefectoral Hospital in Guinea. International Journal of Neurologic Physical Therapy. Vol. 5, No. 2, 2021, pp. 21-24. doi: 10.11648/j.cnn.20210502.14

Received: March 8, 2021; Accepted: March 22, 2021; Published: May 8, 2021

\begin{abstract}
Introduction: Cerebral malaria is one of the spontaneously fatal clinical manifestations of malaria. The aim of our study was to describe the clinical aspects and the evolving profile of cerebral malaria in children at the Coyah prefectural hospital. Material and methods: This was a prospective study of descriptive type over a period of 6 months from July $1^{\text {st }}$ to December $31^{\text {st }}$, 2015. Our study was based on children admitted for severe malaria, presenting neurological signs and responding to our selection criteria. Results: 156 children were collected, with hospital frequency of $46 \%$. The average age was 4.28 years old and the most affected age group was between 0 and 5 years $(80.13 \%)$. The sex ratio=1.33; seizures and coma often preceded by fever were present in 51.92 and $23.72 \%$ respectively. Neuropaludism associated with anemia followed by cerebral malaria associated with hypoglycemia represented $52 \%$ and $26 \%$, respectively, of the clinical phenotypes encountered in our study, while isolated cerebral malaria represented only $14 \% .86 .53 \%$ of patients had a favorable outcome, of which $76.27 \%$ presented no neurological abnormality on discharge and $10.26 \%$ with neurological sequelae on discharge from the hospital. 21 children or $13.47 \%$ died. Discussion: The delay in consultation in an adequate health structure linked on the one hand to the perception of modern medicine in rural areas and on the other hand to the epidemiological context of EBOLA virus hemorrhagic fever was aggravating causes of the diagnosis. Conclusion: Cerebral malaria is a major public health concern. The associated forms frequently observed lead to a fatal outcome because of the difficulties of care, especially in rural areas.
\end{abstract}

Keywords: Neuropaludism, Cerebral Malaria, Prefectoral Hospital, Guinea

\section{Introduction}

Cerebral malaria $(\mathrm{PN})$ is, together with severe anemia, the most common forms of severe malaria [1]. It is one of the spontaneously fatal clinical manifestations of malaria. According to the World Health Organization (WHO), it is an acute febrile encephalopathy caused by Plasmodium Falciparum, and which is characterized by the sequestration of parasitized red blood cells within the micro-vessels of the brain. Cerebral malaria is a real diagnostic and therapeutic emergency, but also a real public health problem. The usual clinical features in children constitute a characteristic triad (coma, convulsion, signs of brainstem distress) in children with anorexia, vomiting, abdominal pain, fever and sometimes cough for 1 to 3 days [2, 3].

The evolution can be favorable, without any abnormality of the neurological examination on discharge, i.e. an ad integrum restitution of the state of consciousness between 24 and 96 hours or unfavorable with neurological sequelae observed on waking in approximately $10 \%$ of cases of unconsciousness or even death. Cerebral malaria occurs in $10 \%$ of pediatric hospitalizations with an estimated case fatality of $18.6 \%$ (3000 
sub-Saharan children die from it every day), mortality in these areas is mainly infantile [2].

\section{Goals}

1) Study the clinical aspects and the evolving profile of cerebral malaria in children at the Coyah prefectural hospital.

2) Determine the hospital frequency of neuropaludism in children at the Coyah prefectural hospital.

3) Describe its different clinical aspects.

4) Describe the fate of patients following hospitalization for neuropaludism at Coyah prefectural hospital.

\section{Materials and Method}

This was a prospective study of a descriptive type, lasting 6 months from July $1^{\text {st }}$ to December $31^{\text {st }}, 2015$. The pediatric department of the Coyah Prefectural Hospital (HPC) served as the study framework. The consultation and hospitalization registers; laboratory records; individual files of hospitalized patients; and a survey sheet established for this purpose were used as supports for the realization of this study.

Patients hospitalized in the pediatric ward during the study period for severe malaria confirmed by thick gout were the target population.

Children 0 to 15 years of age hospitalized for neurological disorders such as coma, delirium or seizures with the presence of thick-gout plasmodium falciparoma during the study period constituted the study population. Our variables were epidemiological; clinics; para clinics; therapeutic and progressive.

\section{Results}

During our study period, 341 patients were admitted to the pediatric ward of Coyah prefectural hospital for severe malaria, of which 156 presented with a clinical picture of cerebral malaria, ie a frequency of $46 \%$; The most common clinical phenotypes encountered in our study were cerebral malaria associated with anemia $(52 \%)$, cerebral malaria associated with hypoglycemia $(26 \%)$, and cerebral malaria isolated (14\%).

The average age of our patients was 4.28 years old with extremes of 1 and 15 years old. The most affected age groups were those of 3 to 5 years $(53.85 \%)$ followed by groups of 0 to 2 years and 6 to 8 years with respective frequencies of $26.28 \%$ and $12.18 \%$. During our study, both sexes were observed with a clear male predominance, ie $57.05 \%$ of cases. The sex ratio is 1.33 . We found a frequency of $30.78 \%$ corresponding to the referral rate of patients from the two most remote rural communes of Coyah prefecture. During our study, the majority of patients were identified during the months of August, September and October with frequencies of $20 \%, 50 \%$, respectively; $25 \%$ and $22.43 \%$. Only 18 patients $(11.54 \%)$ consulted in the first days of disease onset during the study period. Fever was the first reason for consulting our patients with $100 \%$ of cases. Seizures and coma accounted for 51.92 and $23.72 \%$ of cases, respectively.

In our study, 52 patients $(33.33 \%)$ consulted within 1 to 2 days; $73(46.80 \%)$ within 3 to 5 days and $13(8.33 \%)$ consulted beyond 5 days. The majority of our patients $(57.04 \%)$ had a hospital stay of 4 to 5 days, ie 89 patients. 30 of them (19.23\%) had more than 5 days of hospitalization.

The outcome was favorable in $86.53 \%(n=135)$ of the patients, among whom $76.27 \%(\mathrm{n}=119)$ were favorable without any neurological sequelae at the discharge examination and $10.26 \%(n=119)=16)$ with neurological sequelae on discharge from hospital. 21 patients died, or $13.47 \%$ of the study population. During our study, the age groups [3-5] years and [0-2] years were the most affected with respectively 84 and 41 patients or 53.84 and $26.28 \%$ respectively.

The cure and death rates are respectively 55.56 and $42.86 \%$ for the [3-5] year group, then 23.70 and $38.09 \%$ for the [0-2] year group. Children over 5 years of age are the least affected, i.e. $20.51 \%(n=32)$ of patients and a cure rate of $87.5 \%(n=28)$.

Only $11.54 \%(n=18)$ patients were admitted within 24 hours of onset of symptoms in hospital.

During our study period, 30.13\% $(n=47)$ of patients presented with a comatose state, indicative of a severe neurological state with a child-friendly Glasgow score of less than 5 in $64.74 \%(n=101)$ of patients.

\section{Discussion}

During our study period, 341 patients were admitted to the pediatric ward of Coyah prefectural hospital for severe malaria, of which 156 presented with a clinical picture of cerebral malaria, ie a frequency of $46 \%$. Our results are far superior to those reported by TCHOKOTEU et al. in Cameroon [4] who found $2.7 \%$ in their study. The variation in endemicity of the disease from one region to another explains on the one hand this difference but also the effectiveness of protection methods. The disparity in the selection criteria used may also explain this difference.

The most common clinical phenotypes encountered in our study are cerebral malaria associated with anemia (52\%), cerebral malaria associated with hypoglycemia (26\%), and isolated cerebral malaria $(14 \%)$. The average age of our patients is 4.28 years with extremes of 1 and 15 years. The most affected age groups are those of 3 to 5 years $(53.85 \%)$ followed by groups of 0 to 2 years and 6 to 8 years with respective frequencies of 26.28 and $12.18 \%$. Our results show that children under 5 are the most affected by the condition with a total frequency of $80.13 \%$. Results similar to those of BISIMWA et al. [5] in the Democratic Republic of Congo who found frequency of $70.00 \%$ of children under 5 years of age suffering from cerebral malaria. During our study, both sexes were observed with a clear male predominance, ie $57.05 \%$ of cases. The sex ratio is 1.33 . TCHOKOTEU et al. in Cameroon [4] and BISIMWA et al. found a female predominance with a sex ratio of 0.89 and 


\subsection{7 respectively.}

We found a frequency of $30.78 \%$ corresponding to the referral rate of patients from the two most remote rural communes of Coyah prefecture. This low attendance could be explained by the difficulties related to means of travel, road infrastructure but also problems related to the acceptance of modern medicine among our most remote rural populations. The epidemiological context linked to the presence of EBOLA virus hemorrhagic fever and the reluctance of the populations to visit hospitals during this period of the EBOLA epidemic are also to be considered.

During our study, the majority of patients were identified during the months of August, September and October with frequencies of 20.50, respectively; 25 and $22.43 \%$. Our results are superimposable on those of D DIARRA [6] who found frequencies of 14.85 respectively; 20.61 and $34.54 \%$ during the same months. These months correspond to periods of heavy rains, a factor in the maintenance of malaria mosquitoes by favorable conditions: heavy rainfall, grass and patches of water [5]. Only 18 patients $(11.54 \%)$ during our study consulted on the same day of the onset of the disease. Results inferior to those of BISIMWA et al. who found 20\% of patients who consulted on the same day. Delayed consultation is significantly associated with a risk of severe malaria [7].

This result can be explained by a lack of awareness. Most families do not know how to identify symptoms of malaria early. The epidemic context of EBOLA virus haemorrhagic fever during the study period is still to be considered. Fever was the first reason for consultation in our study with $100 \%$ of cases. D DIARRA and BISIMWA et al. found a similar frequency.

Seizures and coma accounted for 51.92 and $23.72 \%$ of cases, respectively. Our results are inferior to those of DIARRA who also found a predominance of convulsions with $70 \%$ of cases and $30 \%$ for coma. Our results differ from those of BISIMWA et al. [5] who found a predominance of coma compared to convulsions with $97.33 \%$ and $90 \%$.

The other neurological signs were marked by restlessness $(17.95 \%)$ and prostration $(10.90 \%)$. BISIMWA et al. found $80 \%$ of agitated patients. OSSOU-NGUIET et al. in Congo found $20.80 \%$ association of cerebral malaria with coma and anemia [7] and SAME EKOBO A et al. [8] in Cameroon described the association of cerebral malaria and hypoglycemia as a biological peculiarity in endemic areas. In Afro-tropical zones, these two associations seem to have a high predictive value for a poor prognosis.

In our study, 52 patients $(33.33 \%)$ consulted within 1 to 2 days; $73(46.80 \%)$ within 3 to 5 days and $13(8.33 \%)$ consulted beyond 5 days. Our results are similar to those of BISIMWA et al. who found a frequency of $44.7 \%$ of patients having consulted within 1 to 2 days. Only 18 patients or $11.54 \%$ were admitted on the same day of the onset of the disease. Results inferior to those of BISIMWA et al. who found $20 \%$ of patients who consulted on the same day. The majority of our patients $(57.04 \%)$ had a hospital stay of 4 to 5 days, ie 89 patients. 30 of them $(19.23 \%)$ had more than 5 days of hospitalization.

During our study, we recorded $86.53 \%(n=135)$ cured patients, of which $76.27 \%(n=119)$ were cured without any neurological sequelae at the discharge examination and $10.26 \%(n=16)$ with neurological sequelae on discharge from hospital. 21 patients died, or $13.47 \%$ of the study population. Our results are consistent with those reported by BISIMWA et al. who find $82 \%$ of cured patients and $15.3 \%$ cases of death. On the other hand, our results differ from those established by TCHOKOTEU et al. who reported $77.8 \%$ cases of cure without neurological sequelae; $16.7 \%$ of cases of cure but with a disturbed neurological examination on discharge from the hospital and $5.6 \%$ of cases of death.

The sequelae encountered were of three types: psychointellectual sequelae (almost all behavioral disorders), motor sequelae (are the ones that go away the fastest) and paroxysmal neurosensory sequelae including 2 cases of postmalaria epileptic seizures and the other manifestations being non-epileptic (especially headaches). B CAMARA et al. [9] reported 1 case of neurological sequelae of severe malaria in children with an epileptic seizure at the Albert Royer University Hospital in Fann in Senegal and recommended that a history of severe malaria such as cerebral malaria be systematically sought during the assessment of the factors. This complication has mainly been reported in children and seems rare in adults according to STRICKER J et al. [10].

During our study, the age groups 3-5 years and 0-2 years were the most affected with 84 and 41 patients respectively, ie 53.84 and $26.28 \%$ respectively.

The cure and death rates are respectively 55.56 and $42.86 \%$ for the $3-5$ year group, then 23.70 and $38.09 \%$ for the [0-2] year group. Our results corroborate those of BISIMWA et al. who found $91.30 \%(n=23)$ deaths among children under 5 years old. Children over 5 years of age are the least affected, i.e. $20.51 \%(n=32)$ of patients and a cure rate of $87.5 \%(n=28)$.

Delay in consultation has been reported in the majority of studies on cerebral malaria in Africa. During our study, $55.77 \%(n=87)$ of the patients showed a delay in the consultation of 3 days or more, marked by a death rate of $71.43 \% \quad(n=15)$. D DIARR reports respective frequencies of 54.2 and $20.61 \%$ for periods of 2 to 3 days and 4 to 7 days. Only $11.54 \%(n=18)$ patients were admitted within 24 hours of onset of symptoms in hospital. Our results are lower than those of BISIMWA et al. who reported $20 \%$ admissions within 24 hours. During our study period, 30.13\% $(n=47)$ of patients presented with a comatose state, indicative of a severe neurological state with a Glasgow score suitable for the child less than 5 in $64.74 \%(n=101)$ of the patients. Our results are much lower than those of TCHOKOTEU et al. as well as those of BISIMWA et al. which respectively reported frequencies of 100 and $97.34 \%$ in Yaoundé in Cameroon and in South Kivu in the Democratic Republic of Congo. However our results corroborate those of Diarra who reported a frequency of $30.00 \%$ in Mali. 


\section{Conclusion}

Cerebral malaria is one of the major spontaneously fatal clinical manifestations and a major cause of infant mortality. The most important clinical phenotypes are the isolated neurological form and the associated forms (cerebral malaria + anemia, cerebral malaria + hypoglycemia or cerebral malaria + respiratory distress) but all lead either to a fatal outcome or to a cure but sometimes with neurological sequelae which may persist in time or not. The clinical course of the infection depends on several factors including the immune status of the infected subject, the time to adequate treatment but also on the treatment which is still insufficient due to the lack of an appropriate technical platform and still qualified personnel. Thus, improving the management of neurological forms of malaria would require a better quality of the technical platform and human resources in order to avoid infant mortality and a cure rate with neurological sequelae which is still very high.

\section{References}

[1] M, SEIDY M, DIOP. S. A, DIOP. B. M, SOW. P. S. Adult cerebral malaria at the infectious diseases clinic at Fann University Hospital in Dakar, Senegal BP 5035 Dakar Sénégal. Clinique. Reçue le 2 avril 2007, accepté le 5 septembre 2007.

[2] TROPICAL MEDICINE; Malaria. News 2015. Professeur Pierre Aubry. 11 / 01/ 2015.

[3] OUATTARA B; Management of cases of cerebral malaria in children from 0 to 15 years old at the Nianan Koro Fomba hospital in Ségou. Doctoral thesis in Medicine. University of Bamako 2007.
[4] TCHOKOTEU P. F, POKA D, EKOBO S. A, NGOGANG J et al.; Childhood cerebral malaria in Yaoundé: clinical, paraclinical and progressive aspect in the pediatric services of the Yaoundé general hospital. BP. 7718, and pediatrics at the Bafoussam Provincial Hospital. Ann. Soc belge Méd. Trop. 1994. 74, 193-202.

[5] BISIMWA MUSHAGALUSA, MANEGABE BABUNGA, MBIYE BADIBANGA, NKONZI KARAZO, KAFUMBA KIBIBI; Childhood cerebral malaria in Lwiro (South Kivu, DRC) International Journal of Innovation and Applied Studies ISSN 2028-9324 Vol. 13 No. 1 Sep 2015, pp. 178-184.

[6] DRAMANE SEKOU DIARRA; Study of mortality and morbidity linked to cerebral malaria in the pediatric department of the Nianan Koro Fomba hospital in Ségou, in children from 6 months to 15 years old. Thèse de Doctorat en Médecine. Université de Bamako 2006.

[7] P. M. OSSOU-NGUIET, A. R. OKOKO, G. EKOUYA BOWASSA, A. P. OKO, J. R. MABIALA-BABELA.; Determinants of cerebral malaria in Congolese pediatric settings. Revue neurologique No 169. 6 Février 2013, pp 510514.

[8] SAME EKOBO A, NGONGANG J, BEDIMO E et al.; Prognostic values of the decrease in antisporozoitic antibodies and hypoglycemia in cerebral malaria. Faculté des sciences de la santé. Yaoundé- Cameroun. Neurologie Tropicale. Ed AUPELF-UREF. John Libbey Eurotext. Paris 1993; pp 369374.

[9] CAMARA B, FAYE P, BA I, AKONDE $\mathrm{F}$ B et al.; Neurological sequelae of severe malaria in children. Bull Soc Pathol Exot 2008; 101, 2, 131-148.

[10] STRICKER J, SAFOURIS S, DIVANO L, STADNIK T et al.; A case of post-cerebral malaria frontal syndrome. Rev Med Brux 2011; 32: 473-6. 